Supporting Information for the article

\title{
Fractional precipitation of amino acids from agro- industrial residues using ethanol
}

Widyarani, Nathan A. Bowden, Ruben C. Kolfschoten, Johan P. M. Sanders, Marieke E. Bruins 
Table S1. Changes of amino acid solubility in a mixture containing two amino acids in water ${ }^{\mathrm{a}}$

\begin{tabular}{|c|c|c|c|c|c|c|c|c|c|c|c|c|}
\hline \multirow{2}{*}{\multicolumn{2}{|c|}{ II }} & \multicolumn{4}{|c|}{ Polar } & \multicolumn{4}{|c|}{ Hydrophobic } & \multicolumn{3}{|c|}{ Special } \\
\hline & & Asn & Ser & Asp & Glu & Leu & Val & Phe & Tyr & Ala & Gly & Cys $2^{b}$ \\
\hline \multirow{4}{*}{$\frac{7}{0}$} & Asn & & & & & & & & & + & + & \\
\hline & Ser & & & + & + & & & & & + & & \\
\hline & Asp & & + & & + & & & & & & + & \\
\hline & Glu & & + & + & & & & & & & + & \\
\hline \multirow{4}{*}{ 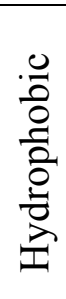 } & Leu & & & & & & $=/-^{\mathrm{c}}$ & & $=$ & - & - & $=$ \\
\hline & Val & & & & & $+/==^{\mathrm{c}}$ & & & & - & & \\
\hline & Phe & & & & & & & & & & $+{ }^{\mathrm{d}}$ & \\
\hline & Tyr & & & & & $=$ & & & & & + & + \\
\hline \multirow{3}{*}{ 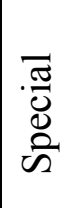 } & Ala & & + & & & - & - & & & & & \\
\hline & Gly & & & + & + & $=$ & & + & $=$ & & & $=$ \\
\hline & ${\text { Cys }{ }^{b}}^{b}$ & & & & & + & $=$ & & + & + & $-1+^{\mathrm{e}}$ & \\
\hline
\end{tabular}

$\mathrm{I}=$ Amino acid that was added first at varying concentrations below its saturation point.

$\mathrm{II}=$ Amino acid that was added to the solution containing amino acid (I) at amounts exceeding its saturation point.

+ Solubility of amino acid (II) increased when concentration of amino acid (I) in the initial solution increased.

- Solubility of amino acid (II) decreased when concentration of amino acid (I) in the initial solution increased.

$=$ Solubility of amino acid (II) did not change when concentration of amino acid (I) in the initial solution increased.

${ }^{a}$ Data compiled from Carta, ${ }^{17}$ Cohn et al. ${ }^{19}$ Grosse Daldrup et al.,${ }^{22}$ Jin and Chao, ${ }^{20}$ Kuramochi et al. ${ }^{23}$ Kurosawa et al.,${ }^{18}$ Soto et al..${ }^{21}$ for L-isomers at $25^{\circ} \mathrm{C}$, except for Ala/Val, Ala/Ser, Asp/Gly, Phe/Gly systems (DL-isomers), Ala/Asn, Ala/Cys2, Val/Cys systems (DL-Ala, DL-Val), Ala/Leu system $\left(30^{\circ} \mathrm{C}\right)$, and $\mathrm{Glu}(\mathrm{I}) / \mathrm{Gly}(\mathrm{II})$ system $\left(60^{\circ} \mathrm{C}\right)$. For amino acids abbreviations see Table $1 ;{ }^{\mathrm{b}} \mathrm{L}$-cystine; ${ }^{\mathrm{c}}$ Result at $25^{\circ} \mathrm{C} /$ result at $30^{\circ} \mathrm{C} ;{ }^{\mathrm{d}}$ Increase then decrease; ${ }^{\mathrm{e}}$ Result from Carta ${ }^{17} /$ result from Cohn et al. ${ }^{19}$ 
Table S2. Amino acid concentration $(\mu \mathrm{mol} / \mathrm{g})$ in the starting mixtures of Protamylasse, experiment at fixed starting material concentration and varying ethanol concentration.

\begin{tabular}{lrrrrrrrrrrr} 
Amino & \multicolumn{10}{c}{ Ecthanol concentration (\%w/w) } \\
\cline { 2 - 11 } Asn & $\mathbf{0}$ & $\mathbf{1 0}$ & $\mathbf{2 0}$ & $\mathbf{3 0}$ & $\mathbf{4 0}$ & $\mathbf{5 0}$ & $\mathbf{6 0}$ & $\mathbf{7 0}$ & $\mathbf{8 0}$ & $\mathbf{9 0}$ & $\mathbf{9 5}$ \\
Gln & 6.8 & 6.7 & 6.9 & 6.8 & 7.0 & 6.8 & 6.9 & 6.8 & 6.9 & 6.9 & 6.9 \\
Ser & 0.0 & 0.0 & 0.0 & 0.0 & 0.0 & 0.0 & 0.0 & 0.0 & 0.0 & 0.0 & 0.0 \\
Thr & 0.6 & 0.6 & 0.6 & 0.6 & 0.7 & 0.6 & 0.6 & 0.6 & 0.6 & 0.6 & 0.7 \\
Asp & 0.3 & 0.3 & 0.3 & 0.3 & 0.3 & 0.3 & 0.3 & 0.3 & 0.3 & 0.3 & 0.3 \\
Glu & 3.5 & 3.4 & 3.5 & 3.5 & 3.6 & 3.5 & 3.5 & 3.5 & 3.5 & 3.5 & 3.5 \\
Arg & 1.6 & 1.5 & 1.6 & 1.6 & 1.6 & 1.6 & 1.6 & 1.6 & 1.6 & 1.6 & 1.6 \\
His & 1.0 & 1.0 & 1.0 & 1.0 & 1.1 & 1.0 & 1.0 & 1.0 & 1.1 & 1.0 & 1.1 \\
Lys & 0.1 & 0.1 & 0.1 & 0.1 & 0.1 & 0.1 & 0.1 & 0.1 & 0.1 & 0.1 & 0.1 \\
Ile & 0.5 & 0.5 & 0.5 & 0.5 & 0.5 & 0.5 & 0.5 & 0.5 & 0.5 & 0.5 & 0.5 \\
Leu & 0.3 & 0.3 & 0.3 & 0.3 & 0.3 & 0.3 & 0.3 & 0.3 & 0.3 & 0.3 & 0.3 \\
Val & 0.2 & 0.2 & 0.2 & 0.2 & 0.2 & 0.2 & 0.2 & 0.2 & 0.2 & 0.2 & 0.2 \\
Phe & 0.9 & 0.9 & 0.9 & 0.9 & 0.9 & 0.9 & 0.9 & 0.9 & 0.9 & 0.9 & 0.9 \\
Trp & 0.3 & 0.3 & 0.3 & 0.3 & 0.3 & 0.3 & 0.3 & 0.3 & 0.3 & 0.3 & 0.3 \\
Tyr & 0.0 & 0.0 & 0.0 & 0.0 & 0.0 & 0.0 & 0.0 & 0.0 & 0.0 & 0.0 & 0.0 \\
Met & 0.3 & 0.3 & 0.3 & 0.3 & 0.3 & 0.3 & 0.3 & 0.3 & 0.3 & 0.3 & 0.3 \\
Pro & 0.1 & 0.1 & 0.1 & 0.1 & 0.1 & 0.1 & 0.1 & 0.1 & 0.1 & 0.1 & 0.1 \\
Ala & 0.3 & 0.3 & 0.3 & 0.3 & 0.3 & 0.3 & 0.3 & 0.3 & 0.3 & 0.3 & 0.3 \\
Gly & 1.5 & 1.5 & 1.5 & 1.5 & 1.5 & 1.5 & 1.5 & 1.5 & 1.5 & 1.5 & 1.5 \\
Cys & 0.2 & 0.2 & 0.2 & 0.2 & 0.2 & 0.2 & 0.2 & 0.2 & 0.2 & 0.2 & 0.2 \\
\hline Total & 0.0 & 0.0 & 0.0 & 0.0 & 0.0 & 0.0 & 0.0 & 0.0 & 0.0 & 0.0 & 0.0 \\
\hline
\end{tabular}


Table S3. Amino acid concentration $(\mu \mathrm{mol} / \mathrm{g})$ in the supernatant of Protamylasse, experiment at fixed starting material concentration and varying ethanol concentration.

\begin{tabular}{lrrrrrrrrrrr} 
Amino & \multicolumn{10}{c}{ Ethanol concentration $\mathbf{( \% w} / \mathbf{w})$} \\
\cline { 2 - 7 } & $\mathbf{0}$ & $\mathbf{1 0}$ & $\mathbf{2 0}$ & $\mathbf{3 0}$ & $\mathbf{4 0}$ & $\mathbf{5 0}$ & $\mathbf{6 0}$ & $\mathbf{7 0}$ & $\mathbf{8 0}$ & $\mathbf{9 0}$ & $\mathbf{9 5}$ \\
Asn & 6.9 & 6.9 & 7.0 & 7.0 & 7.2 & 6.9 & 6.6 & 4.0 & 1.9 & 0.7 & 0.1 \\
Gln & 0.0 & 0.0 & 0.0 & 0.0 & 0.0 & 0.0 & 0.0 & 0.0 & 0.0 & 0.0 & 0.0 \\
Ser & 0.7 & 0.6 & 0.7 & 0.7 & 0.7 & 0.7 & 0.6 & 0.4 & 0.2 & 0.1 & 0.0 \\
Thr & 0.2 & 0.2 & 0.2 & 0.2 & 0.2 & 0.2 & 0.2 & 0.2 & 0.1 & 0.1 & 0.0 \\
Asp & 3.6 & 3.5 & 3.6 & 3.6 & 3.7 & 3.5 & 3.3 & 1.7 & 0.4 & 0.1 & 0.0 \\
Glu & 1.6 & 1.6 & 1.6 & 1.6 & 1.6 & 1.6 & 1.6 & 1.1 & 0.5 & 0.2 & 0.0 \\
Arg & 1.1 & 1.0 & 1.1 & 1.1 & 1.1 & 1.1 & 0.9 & 0.5 & 0.3 & 0.2 & 0.0 \\
His & 0.1 & 0.1 & 0.2 & 0.2 & 0.2 & 0.1 & 0.1 & 0.1 & 0.1 & 0.0 & 0.0 \\
Lys & 0.5 & 0.5 & 0.5 & 0.5 & 0.5 & 0.5 & 0.4 & 0.2 & 0.1 & 0.0 & 0.0 \\
Ile & 0.3 & 0.3 & 0.3 & 0.3 & 0.3 & 0.3 & 0.3 & 0.3 & 0.3 & 0.3 & 0.1 \\
Leu & 0.2 & 0.2 & 0.2 & 0.2 & 0.2 & 0.2 & 0.2 & 0.2 & 0.2 & 0.2 & 0.1 \\
Val & 0.9 & 0.9 & 0.9 & 0.9 & 0.9 & 0.9 & 0.9 & 0.9 & 0.8 & 0.7 & 0.2 \\
Phe & 0.3 & 0.3 & 0.3 & 0.3 & 0.3 & 0.3 & 0.3 & 0.3 & 0.3 & 0.2 & 0.1 \\
Trp & 0.0 & 0.0 & 0.0 & 0.0 & 0.0 & 0.0 & 0.0 & 0.0 & 0.0 & 0.0 & 0.0 \\
Tyr & 0.5 & 0.4 & 0.4 & 0.4 & 0.4 & 0.4 & 0.4 & 0.3 & 0.3 & 0.2 & 0.0 \\
Met & 0.1 & 0.1 & 0.1 & 0.1 & 0.1 & 0.1 & 0.1 & 0.1 & 0.1 & 0.1 & 0.0 \\
Pro & 0.3 & 0.2 & 0.3 & 0.3 & 0.3 & 0.3 & 0.3 & 0.3 & 0.3 & 0.2 & 0.0 \\
Ala & 1.6 & 1.6 & 1.6 & 1.6 & 1.6 & 1.6 & 1.6 & 1.3 & 1.1 & 0.7 & 0.1 \\
Gly & 0.1 & 0.1 & 0.1 & 0.1 & 0.1 & 0.1 & 0.1 & 0.1 & 0.0 & 0.0 & 0.0 \\
Cys & 0.1 & 0.1 & 0.1 & 0.0 & 0.0 & 0.0 & 0.0 & 0.0 & 0.0 & 0.0 & 0.0 \\
\hline Total & $\mathbf{1 9 . 1}$ & $\mathbf{1 8 . 7}$ & $\mathbf{1 9 . 1}$ & $\mathbf{1 9 . 2}$ & $\mathbf{1 9 . 5}$ & $\mathbf{1 9 . 0}$ & $\mathbf{1 8 . 1}$ & $\mathbf{1 2 . 1}$ & $\mathbf{7 . 1}$ & $\mathbf{4 . 0}$ & $\mathbf{0 . 8}$ \\
\hline
\end{tabular}


Table S4. Amino acid concentration $(\mu \mathrm{mol} / \mathrm{g})$ in the starting mixtures of RSP enzymatic hydrolysate, experiment at fixed starting material concentration and varying ethanol concentration.

\begin{tabular}{|c|c|c|c|c|c|c|c|c|c|c|c|}
\hline \multirow{2}{*}{$\begin{array}{c}\text { Amino } \\
\text { acid }\end{array}$} & \multicolumn{11}{|c|}{ Ethanol concentration ( $\% \mathrm{w} / \mathrm{w})$} \\
\hline & $\mathbf{0}$ & 10 & 20 & 30 & 40 & 50 & 60 & 70 & 80 & 90 & 95 \\
\hline Asn & 0.8 & 0.8 & 0.8 & 0.8 & 0.8 & 0.8 & 0.8 & 0.8 & 0.8 & 0.8 & 0.8 \\
\hline Gln & 1.1 & 1.1 & 1.1 & 1.1 & 1.1 & 1.1 & 1.1 & 1.1 & 1.1 & 1.1 & 1.1 \\
\hline Ser & 0.8 & 0.8 & 0.8 & 0.8 & 0.8 & 0.8 & 0.8 & 0.8 & 0.8 & 0.8 & 0.8 \\
\hline Thr & 0.5 & 0.5 & 0.5 & 0.5 & 0.5 & 0.5 & 0.5 & 0.5 & 0.5 & 0.5 & 0.5 \\
\hline Asp & 0.5 & 0.5 & 0.5 & 0.5 & 0.5 & 0.5 & 0.5 & 0.5 & 0.5 & 0.5 & 0.5 \\
\hline Glu & 1.0 & 1.0 & 1.0 & 1.0 & 1.0 & 1.0 & 1.0 & 1.0 & 1.0 & 1.0 & 1.0 \\
\hline Arg & 0.1 & 0.1 & 0.1 & 0.1 & 0.1 & 0.1 & 0.1 & 0.1 & 0.1 & 0.1 & 0.1 \\
\hline His & 0.4 & 0.4 & 0.4 & 0.4 & 0.4 & 0.4 & 0.4 & 0.4 & 0.4 & 0.4 & 0.4 \\
\hline Lys & 0.6 & 0.6 & 0.6 & 0.6 & 0.6 & 0.6 & 0.6 & 0.6 & 0.6 & 0.6 & 0.6 \\
\hline Ile & 1.4 & 1.4 & 1.4 & 1.4 & 1.4 & 1.4 & 1.4 & 1.4 & 1.4 & 1.4 & 1.4 \\
\hline Leu & 3.2 & 3.2 & 3.2 & 3.2 & 3.2 & 3.2 & 3.2 & 3.2 & 3.2 & 3.2 & 3.2 \\
\hline Val & 4.1 & 4.1 & 4.1 & 4.1 & 4.1 & 4.1 & 4.1 & 4.1 & 4.1 & 4.1 & 4.1 \\
\hline Phe & 1.4 & 1.4 & 1.4 & 1.4 & 1.4 & 1.4 & 1.4 & 1.4 & 1.4 & 1.4 & 1.4 \\
\hline $\operatorname{Trp}$ & 0.4 & 0.4 & 0.4 & 0.4 & 0.4 & 0.4 & 0.4 & 0.4 & 0.4 & 0.4 & 0.4 \\
\hline Tyr & 0.1 & 0.1 & 0.1 & 0.1 & 0.1 & 0.1 & 0.1 & 0.1 & 0.1 & 0.1 & 0.1 \\
\hline Met & 0.5 & 0.5 & 0.5 & 0.5 & 0.5 & 0.5 & 0.5 & 0.5 & 0.5 & 0.5 & 0.5 \\
\hline Pro & 1.1 & 1.1 & 1.1 & 1.1 & 1.1 & 1.1 & 1.1 & 1.1 & 1.1 & 1.1 & 1.1 \\
\hline Ala & 1.8 & 1.8 & 1.8 & 1.8 & 1.8 & 1.8 & 1.8 & 1.8 & 1.8 & 1.8 & 1.8 \\
\hline Gly & 0.7 & 0.7 & 0.7 & 0.7 & 0.7 & 0.7 & 0.7 & 0.7 & 0.7 & 0.7 & 0.7 \\
\hline Cys & 0.1 & 0.1 & 0.1 & 0.1 & 0.1 & 0.1 & 0.1 & 0.1 & 0.1 & 0.1 & 0.1 \\
\hline Total & 20.6 & 20.5 & 20.5 & 20.6 & 20.5 & 20.5 & 20.6 & 20.5 & 20.6 & 20.5 & 20.5 \\
\hline
\end{tabular}


Table S5. Amino acid concentration $(\mu \mathrm{mol} / \mathrm{g})$ in the supernatant of RSP enzymatic hydrolysate, experiment at fixed starting material concentration and varying ethanol concentration.

\begin{tabular}{|c|c|c|c|c|c|c|c|c|c|c|c|}
\hline \multirow{2}{*}{$\underset{\text { acid }}{\text { Amino }}$} & \multicolumn{11}{|c|}{ Ethanol concentration $(\% \mathrm{w} / \mathrm{w})$} \\
\hline & $\mathbf{0}$ & 10 & 20 & 30 & 40 & 50 & 60 & 70 & 80 & 90 & 95 \\
\hline Asn & 0.8 & 0.8 & 0.9 & 0.8 & 0.8 & 0.8 & 0.8 & 0.8 & 0.6 & 0.3 & 0.2 \\
\hline Gln & 1.1 & 1.1 & 1.1 & 1.1 & 1.1 & 1.1 & 1.1 & 1.0 & 0.9 & 0.7 & 0.5 \\
\hline Ser & 0.8 & 0.8 & 0.8 & 0.7 & 0.8 & 0.7 & 0.7 & 0.7 & 0.6 & 0.4 & 0.2 \\
\hline Thr & 0.5 & 0.5 & 0.5 & 0.5 & 0.5 & 0.5 & 0.5 & 0.4 & 0.5 & 0.4 & 0.3 \\
\hline Asp & 0.5 & 0.5 & 0.5 & 0.5 & 0.5 & 0.5 & 0.5 & 0.5 & 0.3 & 0.1 & 0.0 \\
\hline Glu & 1.0 & 1.0 & 1.0 & 1.0 & 1.0 & 1.0 & 1.0 & 1.0 & 0.8 & 0.4 & 0.2 \\
\hline Arg & 0.0 & 0.0 & 0.0 & 0.0 & 0.0 & 0.0 & 0.0 & 0.0 & 0.0 & 0.0 & 0.0 \\
\hline His & 0.4 & 0.4 & 0.4 & 0.4 & 0.4 & 0.4 & 0.4 & 0.4 & 0.3 & 0.3 & 0.2 \\
\hline Lys & 0.6 & 0.6 & 0.6 & 0.6 & 0.6 & 0.6 & 0.6 & 0.5 & 0.3 & 0.2 & 0.1 \\
\hline Ile & 1.4 & 1.4 & 1.4 & 1.4 & 1.4 & 1.4 & 1.4 & 1.3 & 1.3 & 1.3 & 1.2 \\
\hline Leu & 3.1 & 3.2 & 3.2 & 3.1 & 3.2 & 3.3 & 3.2 & 3.2 & 3.2 & 3.1 & 3.0 \\
\hline Val & 4.0 & 4.1 & 4.1 & 3.9 & 4.0 & 4.0 & 3.9 & 3.8 & 3.7 & 3.6 & 3.4 \\
\hline Phe & 1.3 & 1.4 & 1.4 & 1.3 & 1.4 & 1.4 & 1.4 & 1.4 & 1.4 & 1.3 & 1.2 \\
\hline Trp & 0.4 & 0.4 & 0.4 & 0.4 & 0.4 & 0.4 & 0.4 & 0.4 & 0.4 & 0.4 & 0.4 \\
\hline Tyr & 0.1 & 0.1 & 0.1 & 0.1 & 0.1 & 0.1 & 0.0 & 0.0 & 0.0 & 0.0 & 0.0 \\
\hline Met & 0.5 & 0.5 & 0.5 & 0.4 & 0.5 & 0.5 & 0.5 & 0.4 & 0.4 & 0.4 & 0.4 \\
\hline Pro & 1.0 & 1.0 & 1.1 & 1.0 & 1.0 & 1.0 & 1.0 & 1.0 & 0.9 & 0.9 & 0.8 \\
\hline Ala & 1.8 & 1.8 & 1.8 & 1.7 & 1.8 & 1.8 & 1.7 & 1.7 & 1.6 & 1.5 & 1.2 \\
\hline Gly & 0.7 & 0.7 & 0.7 & 0.6 & 0.7 & 0.7 & 0.6 & 0.6 & 0.5 & 0.4 & 0.3 \\
\hline Cys & 0.1 & 0.1 & 0.1 & 0.1 & 0.1 & 0.1 & 0.1 & 0.1 & 0.1 & 0.1 & 0.0 \\
\hline Total & 20.1 & 20.5 & 20.5 & 19.6 & 20.1 & 20.3 & 19.8 & 19.2 & 17.9 & 15.6 & 13.8 \\
\hline
\end{tabular}


Table S6. Amino acid concentration $(\mu \mathrm{mol} / \mathrm{g})$ in the starting mixtures of Protamylasse, experiment at varying starting material concentration and fixed $(60 \% \mathrm{w} / \mathrm{w})$ ethanol concentration.

\begin{tabular}{|c|c|c|c|c|c|}
\hline \multirow{2}{*}{ Amino acid } & \multicolumn{5}{|c|}{ Protamylasse concentration $(\% \mathrm{w} / \mathrm{w})$} \\
\hline & 5 & 10 & 20 & 30 & 40 \\
\hline Asparagine & 7 & 14 & 27 & 41 & 54 \\
\hline Glutamine & 0 & 0 & 0 & 0 & 0 \\
\hline Serine & 1 & 1 & 3 & 4 & 5 \\
\hline Threonine & 0 & 1 & 1 & 2 & 3 \\
\hline Aspartic acid & 4 & 7 & 14 & 21 & 28 \\
\hline Glutamic acid & 2 & 3 & 6 & 9 & 12 \\
\hline Arginine & 1 & 2 & 4 & 6 & 8 \\
\hline Histidine & 0 & 0 & 1 & 1 & 1 \\
\hline Lysine & 0 & 1 & 2 & 3 & 4 \\
\hline Isoleucine & 0 & 1 & 1 & 2 & 3 \\
\hline Leucine & 0 & 0 & 1 & 1 & 2 \\
\hline Valine & 1 & 2 & 3 & 5 & 7 \\
\hline Phenylalanine & 0 & 1 & 1 & 2 & 2 \\
\hline Tryptophan & 0 & 0 & 0 & 0 & 0 \\
\hline Tyrosine & 0 & 1 & 1 & 2 & 2 \\
\hline Methionine & 0 & 0 & 0 & 1 & 1 \\
\hline Proline & 0 & 1 & 1 & 2 & 2 \\
\hline Alanine & 2 & 3 & 6 & 9 & 12 \\
\hline Glycine & 0 & 0 & 1 & 1 & 1 \\
\hline Cysteine & 0 & 0 & 0 & 0 & 0 \\
\hline Polar uncharged & 8 & 15 & 31 & 46 & 62 \\
\hline Negative & 5 & 10 & 20 & 30 & 40 \\
\hline Positive & 2 & 3 & 7 & 10 & 13 \\
\hline Hydrophobic & 2 & 5 & 9 & 14 & 19 \\
\hline Special & 2 & 3 & 7 & 10 & 13 \\
\hline Total & 19 & 37 & 73 & 110 & 147 \\
\hline
\end{tabular}


Table S.7 Amino acid concentration $(\mu \mathrm{mol} / \mathrm{g})$ in the supernatant of Protamylasse, experiment at varying starting material concentration and fixed $(60 \% \mathrm{w} / \mathrm{w})$ ethanol concentration.

\begin{tabular}{lrrrrr} 
& \multicolumn{5}{c}{ Protamylasse concentration (\%w/w) } \\
\cline { 2 - 6 } Amino acid & $\mathbf{5}$ & $\mathbf{1 0}$ & $\mathbf{2 0}$ & $\mathbf{3 0}$ & $\mathbf{4 0}$ \\
Asparagine & 6.6 & 8.9 & 8.5 & 6.2 & 3.9 \\
Glutamine & 0.0 & 0.0 & 0.0 & 0.0 & 0.0 \\
Serine & 0.6 & 0.8 & 0.8 & 0.7 & 0.4 \\
Threonine & 0.2 & 0.4 & 0.5 & 0.5 & 0.4 \\
Aspartic acid & 3.3 & 4.0 & 2.8 & 1.4 & 0.7 \\
Glutamic acid & 1.6 & 2.1 & 2.0 & 1.3 & 0.7 \\
Arginine & 0.9 & 1.2 & 1.4 & 1.4 & 1.1 \\
Histidine & 0.1 & 0.1 & 0.1 & 0.1 & 0.1 \\
Lysine & 0.4 & 0.4 & 0.4 & 0.3 & 0.3 \\
Isoleucine & 0.3 & 0.6 & 1.3 & 1.8 & 2.3 \\
Leucine & 0.2 & 0.5 & 0.9 & 1.4 & 1.7 \\
Valine & 0.9 & 1.6 & 3.0 & 4.1 & 4.6 \\
Phenylalanine & 0.3 & 0.6 & 1.1 & 1.6 & 1.9 \\
Tryptophan & 0.0 & 0.0 & 0.0 & 0.1 & 0.1 \\
Tyrosine & 0.4 & 0.4 & 1.0 & 1.2 & 1.2 \\
Methionine & 0.1 & 0.3 & 0.5 & 0.6 & 0.7 \\
Proline & 0.3 & 0.6 & 1.1 & 1.3 & 1.3 \\
Alanine & 1.6 & 2.6 & 3.7 & 3.9 & 3.4 \\
Glycine & 0.1 & 0.2 & 0.2 & 0.1 & 0.1 \\
Cysteine & 0.0 & 0.0 & 0.1 & 0.1 & 0.1 \\
\hline Polar uncharged & 7 & 10 & 10 & 7 & 5 \\
Negative & 5 & 6 & 5 & 3 & 1 \\
Positive & 1 & 2 & 2 & 2 & 1 \\
Hydrophobic & 3 & 5 & 9 & 12 & 14 \\
Special & 2 & 3 & 4 & 4 & 4 \\
\hline Total & $\mathbf{1 8}$ & $\mathbf{2 5}$ & $\mathbf{3 0}$ & $\mathbf{2 8}$ & $\mathbf{2 5}$ \\
\hline & & & & & \\
\hline & 0.3 &
\end{tabular}

